\title{
Modification of the surface of activated carbon electrodes for capacitive mixing energy extraction from salinity differences
}

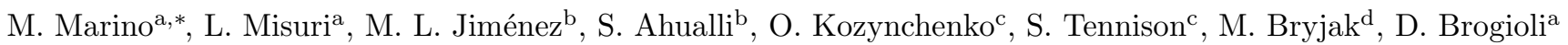 \\ ${ }^{a}$ Dipartimento di Scienze della Salute, Università degli Studi di Milano - Bicocca, via Cadore 48, Monza (MB) 20900, Italy \\ ${ }^{b}$ Department of Applied Physics, School of Sciences, University of Granada, 18071, Granada, Spain \\ ${ }^{c}$ MAST Carbon International Ltd. of Jays Close, Basingstoke, Hampshire, RG22 4BA, United Kingdom \\ ${ }^{d}$ Politechnika Wrocklawska, Wybrzéze Wyspiánskiego 27, 50-370 Wroctaw, Poland
}

\begin{abstract}
The "capacitive mixing" (CAPMIX) is one of the techniques aimed at the extraction of energy from the salinity difference between sea and rivers. It is based on the rise of the voltage between two electrodes, taking place when the salt concentration of the solution in which they are dipped is changed. We study the rise of the potential of activated carbon electrodes in $\mathrm{NaCl}$ solutions, as a function of their charging state. We evaluate the effect of the modification of the materials obtained by adsorption of charged molecules. We observe a displacement of the potential at which the potential rise vanishes, as predicted by the electric double layer theories. Moreover, we observe a saturation of the potential rise at high charging states, to a value that is nearly independent of the analyzed material. This saturation represents the most relevant element that determines the performances of the CAPMIX cell under study; we attribute it to a kinetic effect.
\end{abstract}

Keywords: Energy from salinity difference, Surface groups, Capacitive mixing, Electric double layer, Gouy-Chapman-Stern model

\section{Introduction}

Naturally occurring salinity differences can be used for generating completely clean and renewable energy $[1,2,3]$. For example, each liter of river water dispersed into the sea corresponds to a free energy loss of around $2.3 \mathrm{~kJ}$, and a significant fraction of this energy could be intercepted and converted into electrical energy. Considering all the rivers, the global potential of this source of energy is around 1 TW [4], a relevant fraction of the whole energy demand. Brines can also be locally available: for example, salt lakes (e.g. Dead Sea) [5], coal-mine brines [6] produced by dissolving geological deposits, or salterns [7]. They can be used versus sea water, thus avoiding the fresh water consumption.

The key point of the above described processes is the conversion of the salinity difference into electrical current. Known techniques include pressure-retarded osmosis (PRO) $[8,9,10]$ and reverse electrodialysis (RED) $[11,12]$. In $\mathrm{PRO}$, a semi-permeable membrane is interposed between

\footnotetext{
*Corresponding author. Tel. +3902 6448 8244; fax: +39026448 8068

Email addresses: massimo.marino@unimib.it (M. Marino), lorenza.misuri@unimib.it (L. Misuri), jimenezo@ugr.es (M. L. Jiménez), sahualli@ugr.es (S. Ahualli), kozynch@mastcarbon.co.uk (O. Kozynchenko),

steve.tennison@mastcarbon.co.uk (S. Tennison), marek.bryjak@gmail.com (M. Bryjak), dbrogioli@gmail.com (D. Brogioli)
}

salt and fresh water, generating an osmotic water flow that is fed to a turbine. In RED, the membranes are permeable to either positive or negative ions; the ion diffusion across them constitutes a current that can be extracted.

A new technique, called "capacitive mixing" (CAPMIX) has been recently introduced $[13,14,15,16,17]$. This technique performs the mixing process of the two solutions in a controlled way, by means of the CAPMIX cycle sketched in Fig. 1a. A cell contains a couple of electrodes, dipped into the ionic solution. The cycle begins with the cell filled with the high-salinity solution. The steps are four:

A The cell is charged by means of an external device.

B The circuit is opened. The solution in the cell is substituted with the low-salinity feed solution.

C The cell is discharged through a load; the electrical current flows in the opposite direction with respect to step A.

D The circuit is opened. The liquid in the cell is substituted with the high-salinity feed solution.

Figure 1b shows the voltage versus charge graph for this cycle. During step A the cell voltage increases, and an electrical charge is temporarily stored in the electrodes. The solution change that takes place in step B, in open circuit, induces a cell voltage rise $\Delta V$. The stored charge 
is recovered in step $\mathrm{C}$, at a higher voltage with respect to step A. For this reason, due to the voltage rise, the curve encloses an area, which represents the extracted energy.

During step A, the ions coming from the solution at higher salinity are temporarily stored into the electrodes, and they are later released into the solution at lower salinity during step $\mathrm{C}$, thus decreasing the total salinity difference. This process can be described as a capacitormediated mixing, hence the name "capacitive mixing". It is thus evident that the energy is extracted at the expense

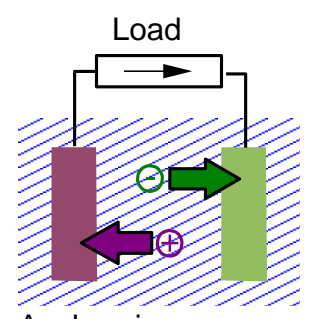

A: charging

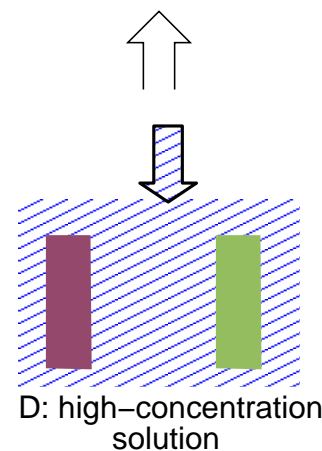

solution
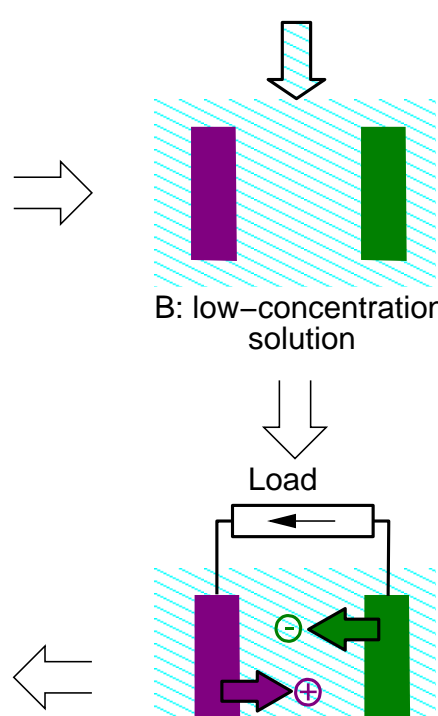

B: low-concentration solution

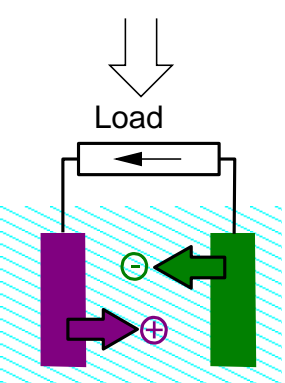

C: discharging b

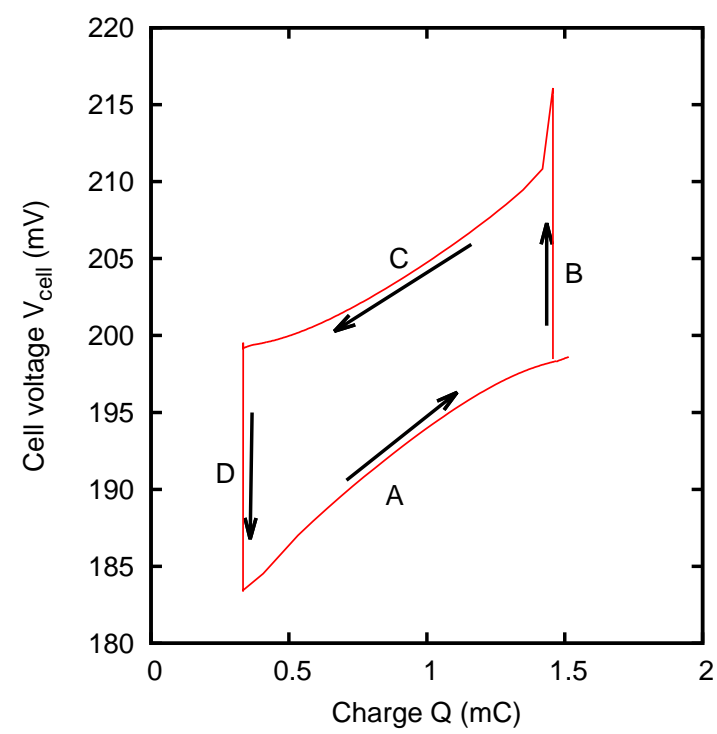

Figure 1: The CAPMIX cycle. Panel a: sketch of the cycle. Panel b: representation of the cycle in the voltage versus charge graph. The phases are: A, charging; B, flow of low-salinity solution; C, discharging; D, flow of high-salinity solution. of the free energy of the solutions; indeed, it has been shown that the voltage rise is connected with the ability of the electrodes to store the salt inside them, when they are charged during phase A [18].

The key element of the CAPMIX technique is the voltage rise that takes place when the solution concentration is changed: indeed, the produced power is roughly proportional to the square of the voltage rise, by assuming a fixed internal resistance. Various techniques, based on different physical principles and making use of different types of electrodes, have been proposed for obtaining the voltage rise:

Capacitive double layer expansion (CDLE) makes use of microporous (typically, activated carbon) electrodes $[19,20,21]$. The voltage rise is due to the variation of the thickness of the electric double layer that forms on the internal surface of the porous electrodes.

Capacitive Donnan potential (CDP) makes use of assemblies, each composed by an activated carbon electrode covered with a perm-selective membrane $[22$, $23,24,25,26]$. The voltage rise is due to the variation of the Donnan potential across the membrane.

Battery-like electrodes make use of materials that capture the ions in the solution by means of redox reactions $[27,28,29,30]$. The voltage of the electrodes with respect to the solution reflects the chemical potential of the ions in the solution, that changes according to the variation of their activity; this originates the voltage rise.

In the seminal work [13], the electric double layers necessary for the CDLE technique were created by electrically charging the electrodes by means of an external power supply. Unfortunately, the self-discharge of the activated carbon led to the necessity of continuously charging the electrodes, thus losing an amount of power that was comparable with the produced power. For this reason, experimental and theoretical investigations have been carried out $[31,32]$ about the possibility of "chemically" charging the electrodes, so as to produce on their surface an electric double layer without the need of an external power supply, and so without undergoing the self-discharge phenomenon. A similar chemical charging has been already studied in the context of capacitive deionization [33].

In this paper, we present results on the open-circuit potentials, either in high- or low-salinity solutions, of activated carbon electrodes that are chemically charged by adsorption or chemical binding of charged molecules on their surface, obtained by means of suitable specific treatments. In Sect. 2 we describe the experimental methods and the preparation of the samples, while in Sect. 3 we present the experimental results. We show that the modification with charged molecules changes the potential rise of the electrodes, and allows us to produce couples of electrodes with improved voltage rise. 
In order to shed light on the physical processes lying behind the observed behavior, we also study the relationship between the potential rise and the base potential, i.e. the potential of the electrode in the high salinity solution, as determined by the electrode's charging status. Various models predict [31] that the potential rise saturates to a constant positive (negative) value for increasing (decreasing) base potential, with a transition between the two at intermediate potentials. Our experimental results show that, in all the samples, it is possible to observe at least one of the two saturations (either at high or low potentials) in the range of base potentials that is accessible by experiments; moreover, a part of the transition zone is also visible. In Sect. 4 we discuss the results and propose qualitative explanations.

\section{Materials and methods}

\subsection{Experimental setup}

Figure 2a shows the experimental setup. The liquid in the cell can be switched between two solutions at different concentrations. The voltages of the two activated carbon electrodes with respect to the reference electrode are monitored; the resulting potentials are called $\varphi(t)$ and $\varphi_{C}(t)$, respectively for the working and counter electrodes (arbitrarily chosen). In the work presented in this paper, all the potentials are measured with respect to a $\mathrm{Ag} / \mathrm{AgCl}$ reference electrode with $3 \mathrm{M}$ concentration of $\mathrm{KCl}$. We also have the possibility to charge one of the electrodes at a given voltage with respect to the reference electrode, by means of a potentiostat, using the other electrode as counter electrode.

\subsection{Definition of electrode potential, potential rise and cell voltage}

The graph in Fig. 2b shows a typical behavior of the potentials $\varphi(t)$ and $\varphi_{C}(t)$ of the two electrodes as functions of time during the salinity change cycle. It can be noticed that the potential $\varphi(t)$ of the working electrode changes as a function of the salinity of the solution in which it is immersed. It asymptotically reaches the values $\varphi^{H}$ and $\varphi^{L}$ respectively in the high- and low-salinity solutions, at concentrations $c_{H}$ and $c_{L}$. The potential in high-salinity solution, i.e. $\varphi^{H}$, will be called the "base potential" in the following, and represents the charging status of the electrode; however, the choice of the high-salinity solution for the definition of the base potential is arbitrary. We will define $\varphi_{C}^{H}$ and $\varphi_{C}^{L}$ analogously, for the counter electrode. The potential rise of an electrode is the difference between the potentials in the dilute and concentrated solutions. The potential rise is thus defined as $\Delta \varphi=\varphi^{L}-\varphi^{H}$ and $\Delta \varphi_{C}=\varphi_{C}^{L}-\varphi_{C}^{H}$, respectively for the working and counter electrodes. We can notice that the two electrodes of Fig. 2b have voltage rises with opposite signs. (a)

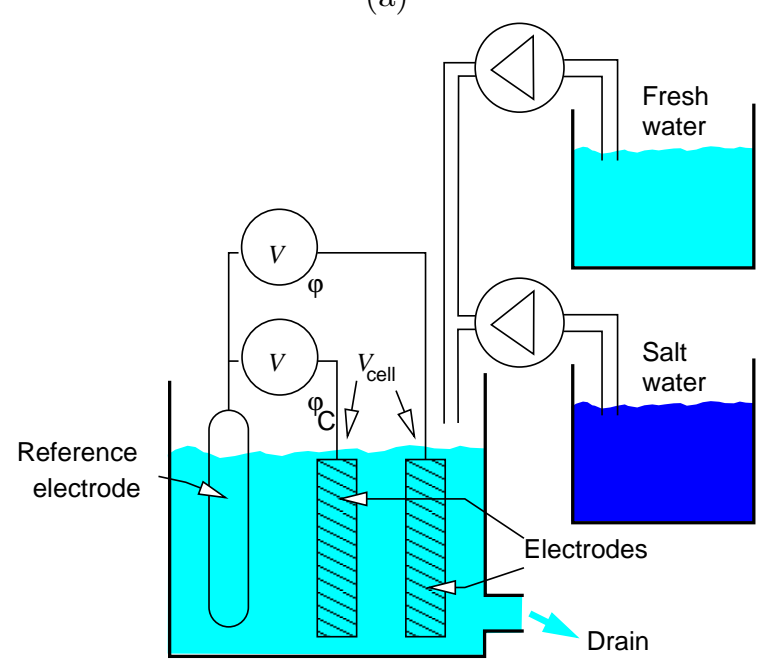

(b)

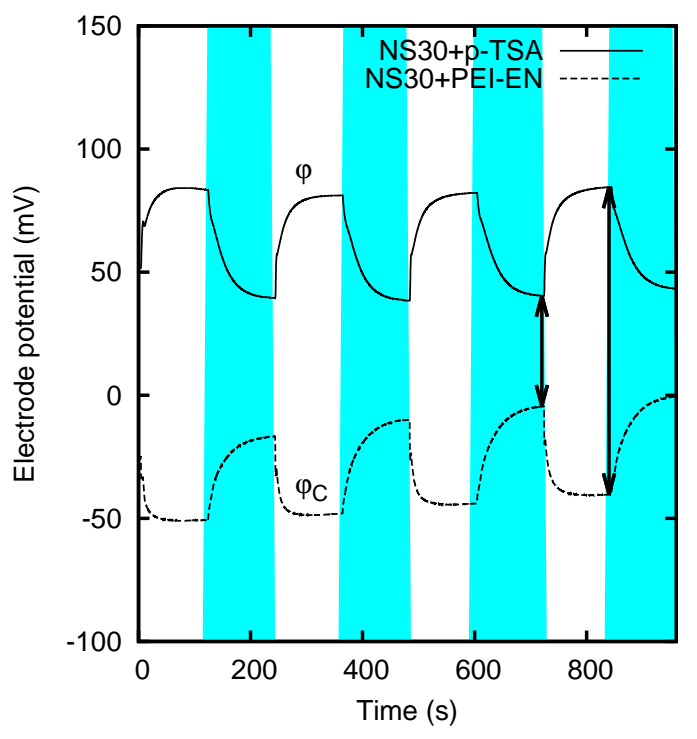

Figure 2: Panel a: experimental setup. Panel b: potentials $\varphi(t)$ and $\varphi_{C}(t)$ of the two electrodes as functions of time during salinity change cycles. The cell voltages $V_{\text {cell }}(t)=\varphi(t)-\varphi_{C}(t)$ in the two different solutions are represented by the arrows. The electrodes are made of NS30 material, respectively functionalized by adsorption of PEI-EN and p-TSA (see Sect. 2.4 for the description of the materials). The salinities of the solutions are $500 \mathrm{mM}$ and $20 \mathrm{mM}$ of $\mathrm{NaCl}$ respectively. The blue shaded areas represent the low-salinity steps of the cycle. 
The cell voltage, defined as $V_{\text {cell }}(t)=\varphi(t)-\varphi_{C}(t)$, also changes as a function of the salinity, with a cell voltage rise

$$
\Delta V_{\text {cell }}=\Delta \varphi-\Delta \varphi_{C}
$$

The cell voltages at low and high salinity are represented by the two arrows in the graph of Fig. 2b. Note that, at variance with the cell represented in Fig. 1, in this case the cell voltage is higher in the high-salinity solution.

Appropriate CAPMIX electrodes require two characteristics: the ability to store charge and the existence of a cell voltage rise $\Delta V_{\text {cell }}$ upon salinity change; in turn, this feature requires that the potential rises $\Delta \varphi$ and $\Delta \varphi_{C}$ of the two electrodes are different, like in the case of the electrodes reported in Fig. 2. It is desirable that one of the electrodes has a positive potential rise and the other a negative potential rise. In the following, we will use the terms "positive-potential-rise" and "negative-potential-rise" electrodes to refer to electrodes with positive (i.e. $\phi^{L}>\phi^{H}$ ) and negative $\left(\phi^{L}<\phi^{H}\right)$ potential rise, respectively; in all the studied cases, this means that they adsorb anions and cations, respectively. The relation between the voltage rise and the salt adsorption has been recently studied [18]. It's worth noting that the notion of positive- and negative-potential-rise does not mean that the electrode is the "positive" or the "negative" electrode of a given couple, like in batteries, nor is it related to the behavior as "anode" or "cathode" in an electrochemical cell.

The produced power is of the order of the square of the cell voltage rise $\Delta V_{\text {cell }}$. For this reason, for CAPMIX we will select couples of electrodes with the largest difference of potential rises $\Delta \varphi-\Delta \varphi_{C}$. This explains the importance of the concept of the potential rise for the CAPMIX technique.

\subsection{Measurement of the potential rise at various base po- tentials}

When an activated carbon electrode is dipped into an ionic water solution, its open-circuit potential takes a characteristic value $\varphi_{S}$, that we call "spontaneous potential". Such electrodes act as supercapacitors: they can accumulate a charge, and their potential changes roughly proportionally to the accumulated charge. The open-circuit potential of the electrode can thus be changed. Nevertheless, if the electrode is left in open circuit for a sufficiently long time, it slowly comes back to its spontaneous potential [32]: it is the well-known self-discharge, which takes place on a time scale of the order of hours. Indeed, in the literature [34] the term "open-circuit potential" is usually only reserved to the spontaneous potential.

The measurement of the potential rise can be performed on the time scale of the minutes, that is much shorter than the time scale over which the self-discharge takes place. By charging the electrode under analysis by means of a potentiostat, it is thus possible to vary its potential in the high salinity solution from the spontaneous value $\varphi_{S}$ to another arbitrary (at least in a certain range) value $\varphi^{H}$, and then measure the potential rise $\Delta \varphi$ in open circuit at that given base potential $\varphi^{H}$.

Experimentally, the measurement of the $\Delta \varphi$ vs. $\varphi^{H}$ curve is performed as follows. The potential of the electrodes is monitored with respect to a reference electrode $(\mathrm{Ag} / \mathrm{AgCl}$ in $3 \mathrm{M} \mathrm{KCl})$. The salinity of the $\mathrm{NaCl}$ solution is switched between $20 \mathrm{mM}$ (typical river water concentration) and $500 \mathrm{mM}$ (typical sea water concentration) every $120 \mathrm{~s}$ and the potential rise is evaluated as the difference between the potentials that are reached at the end of each step. We also pay attention to subtract from the measurements a possible drift of the potential due to self-discharge, which as we have explained is however small on such short time-scales.

The base potential of the electrodes just dipped into the solutions is assumed to be the spontaneous potential. Different base potentials are obtained by charging the electrodes for 30 minutes at a constant potential with respect to the reference electrode by means of the potentiostat.

As the base potential is progressively displaced from the spontaneous potential, the effect of the self-discharge becomes increasingly evident [32]. The self-discharge becomes particularly relevant below $-100 \mathrm{mV}$ and above $600 \mathrm{mV}$. The presence of the self-discharge prevents measurements outside this range. A description of various Faradaic reactions that are likely to be involved in the self-discharge process on the surface of the activated carbon can be found in the review [35] (see section 4.4.2 and figure 9 thereof), in the context of capacitive deionization.

By observing the $\Delta \varphi$ vs. $\varphi^{H}$ curve of an activated carbon, it can often be noticed that it intersects the horizontal axis $\Delta \varphi=0$ at some value $\varphi^{H}=\varphi^{0}$. We will call $\varphi^{0}$ the "potential of zero-rise" (PZR). If the potential rise is due to the electric double layer dynamics, the PZR $\varphi^{0}$ correspond to the potential at which the diffuse part of the double layer disappears, and does not necessarily correspond to the potential of zero charge [31].

\subsection{Materials}

The electrodes we analyzed are $1 \times 1 \mathrm{~cm}$ graphite foils, constituting the current collector, covered with a $100 \mu \mathrm{m}-$ thick film. In the experiments, we used the following commercially available activated carbon materials, specifically designed for supercapacitors:

\section{NS30 Norit DLC Super 30 [36];}

YP-50F Kuraray Chemical Co. steam activated coconut carbon [37].

The NS30 films were first prepared as follows. A slurry is obtained by mixing $90 \%$ of activated carbon powder and $10 \%$ polyvinylidene fluoride (PVDF) as a binder, plus NMethyl-2-pyrrolidone as solvent. The slurry is then cast on the current collector by doctor blade technique. After drying, the films are approximately $100 \mu \mathrm{m}$ thick, with a mass of approximately $10 \mathrm{mg} / \mathrm{cm}^{3}$. 
After casting, some of the NS30 films were chemically functionalized by adsorption of either p-toluenesulfonic acid (p-TSA, a negatively charged molecule) or polyethyleneimine branched with ethylene diamine (PEI-EN, a positively charged polymer), using the following methods. In the first case, the current collector with the NS30 film is dipped into an aqueous solution with $50 \%$ p-TSA. The solution is heated in a boiling water bath until solution temperature reaches $90^{\circ} \mathrm{C}$ and left for spontaneous cooling overnight. The modified electrode is then rinsed with deionized water and dried at ambient conditions. The modification with PEIEN is instead carried out by brush-coating of the NS30 film with $30 \%$ aqueous PEI-EN solution followed by drying in the oven at $110^{\circ} \mathrm{C}$ for one hour. The resulting films are named "NS30", "NS30+p-TSA" and "NS30+PEI-EN" respectively for the unmodified material and for the material with adsorbed p-TSA and PEI-EN.

One of the slurries used for preparing the YP-50F films was composed by $80 \%$ activated carbon powder, $10 \% \mathrm{TiO}_{2}$ powder and $10 \%$ polyvinyl chloride as binder. The second slurry was composed by $90 \%$ activated carbon powder and $10 \%$ chitosan as binder. Chitosan in water has positively charged groups $\mathrm{NH}_{3}^{+}$, and we assume that it is partially adsorbed in the activated carbon particles during the preparation of the sample. The slurries were then cast on the graphite current collector by doctor blade technique. After drying, the films are approximately $100 \mu \mathrm{m}$ thick. The obtained films will be named respectively "YP$50 \mathrm{~F}+\mathrm{TiO} 2$ " and "YP-50F+chitosan". We can argue that, from our point of view, the main difference between them is that the latter contains adsorbed chitosan, and so positively charged molecules, which are instead absent in the former.

\section{Experimental results}

The open circuit potentials, during salinity change cycles, of three different materials are shown in Fig. 3. The graphs show the base potential $\varphi^{H}$ and the potential rise $\Delta \varphi$. It can be noticed that the materials have different base potentials and potential rises.

Figure 4 shows the measured potential rises of the two materials without adsorbed molecules, i.e. NS30 and YP$50 \mathrm{~F}+\mathrm{TiO} 2$, at their spontaneous potentials and in charged conditions. It can be noticed that the potential rise changes as a function of the base potential, and thus of the charge accumulated in the electrode. A nearly linear dependence can be observed in a quite wide range of base potentials; the slope is similar for the two materials. For $\phi_{H}$ approaching $500 \mathrm{mV}$, the potential rise of NS30 seems to present a saturation at approximately $45 \mathrm{mV}$. The last points near the right margin of the graph suggest that also YP-50F+TiO2 may display a similar behavior. The curves appear translated along the horizontal axis and thus the $\operatorname{PZR} \varphi^{0}$ is different for the two materials. It must be emphasized that the base voltage range that is shown in the figure is limited by the stability of the electrodes in water.
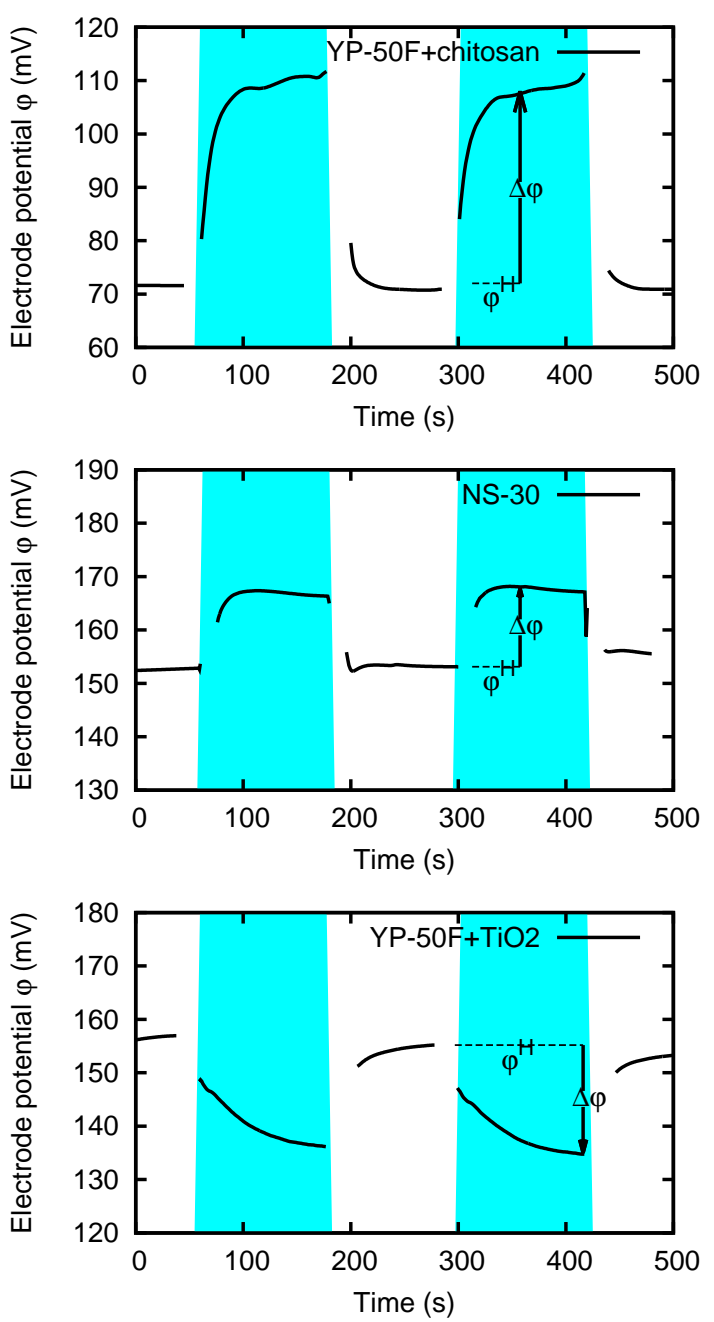

Figure 3: Dependence of the open-circuit potential $\varphi$ of three different electrodes on the salinity of the solution in which they are immersed. The materials are, from top to bottom: YP-50F+chitosan, NS30, and YP-50F+TiO2, see Sect. 2.4 for the description. The salinities of solutions are $500 \mathrm{mM}$ and $20 \mathrm{mM}$ of $\mathrm{NaCl}$. The blue shaded areas represent the low-salinity steps of the cycle. 


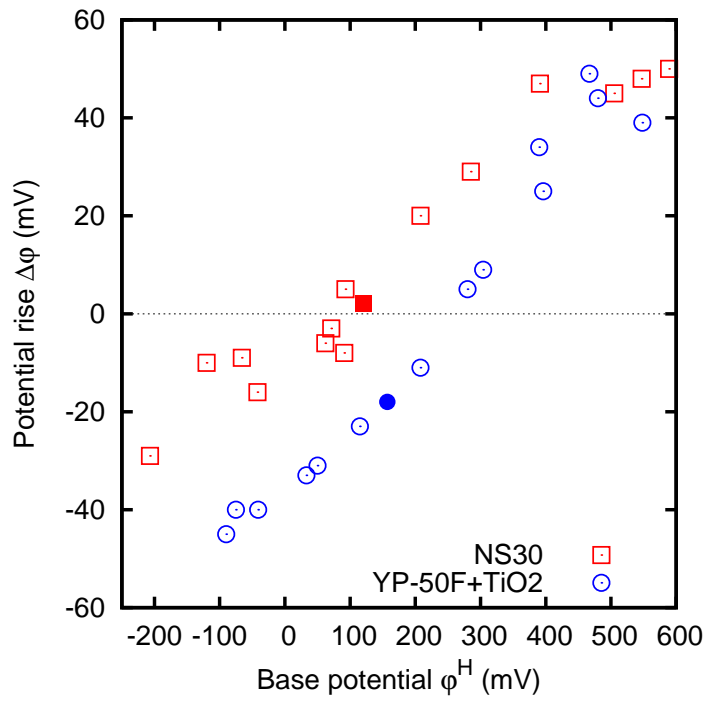

Figure 4: Experimentally measured potential rise $\Delta \varphi$ of the two samples of activated carbon without adsorbed molecules (see Sect. 2 for the description) in sodium chloride at $c_{H}=500 \mathrm{mM}$ and $c_{L}=20 \mathrm{mM}$, at various base potentials $\varphi^{H}$. The solid symbols represent the measurements at the spontaneous potential $\varphi_{S}$.

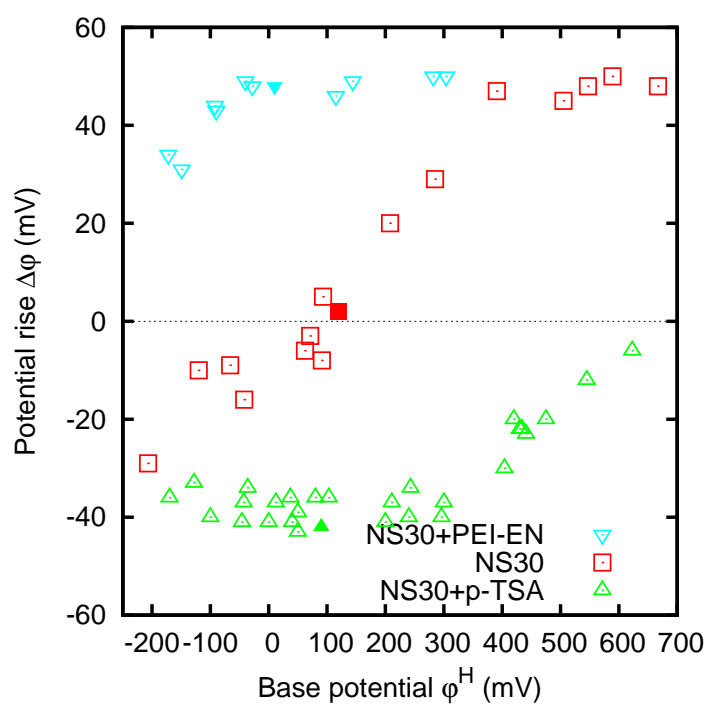

Figure 5: Experimentally measured potential rise $\Delta \varphi$ of NS30 activated carbon electrodes modified by adsorption of charged molecules, p-TSA and PEI-EN (see Sect. 2 for the description) in the same conditions of Fig. 4. The solid symbols refer to the materials at their spontaneous potentials.

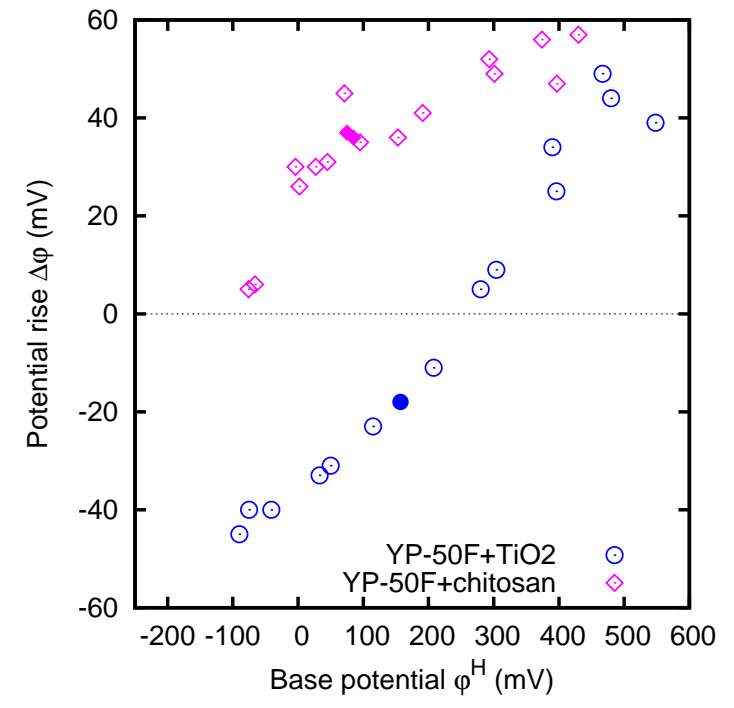

Figure 6: Experimentally measured potential rise $\Delta \varphi$ of the two YP-50F activated carbon electrodes, either with $\mathrm{TiO}_{2}$ or modified by the adsorption of part of the binder chitosan (see Sect. 2 for the description), in the same conditions of Fig. 4. The solid symbols refer to the materials at their spontaneous potentials.

Figure 5 shows the behavior of the unmodified NS30 material, and of the same material modified by adsorption of charged molecules, i.e. p-TSA and PEI-EN. As in the case of Fig. 4, the potential rise depends on the base potential. The linear dependence can be observed for the $\mathrm{NS} 30+\mathrm{p}-\mathrm{TSA}$ sample in the range from 300 to $700 \mathrm{mV}$, and can be also argued for the NS30+PEI-EN in the range from -200 to $-50 \mathrm{mV}$, with slopes that are roughly similar to that of the NS30 material.

The plateau shown by the NS30+PEI-EN at base potentials above $-50 \mathrm{mV}$ is similar to the saturation at high potential that was already noticed in Fig. 4. Analogously, the NS30+p-TSA material presents a clear saturation at base potentials lower than $300 \mathrm{mV}$; this saturation seems to take place at approximately $-40 \mathrm{mV}$.

It can be noticed that the PZR of the sample with pTSA is translated to the right with respect to the NS30 without adsorbed molecules, while it can be argued that it is translated to the left for the sample with PEI-EN.

Figure 6 shows the behavior of the YP-50F material, either with $\mathrm{TiO}_{2}$ or with adsorption of chitosan. As in the case of Fig. 4, the potential rise depends on the base potential. Also in the case of the adsorbed chitosan, a roughly linear dependence can be observed in the range from -100 to $200 \mathrm{mV}$; the slope is roughly the same as that of NS30 or YP-50F without adsorbed molecules. Moreover, the slope clearly decreases for base potentials above $50 \mathrm{mV}$, suggesting that a saturation of the potential rise is likely to take place also for this material. A translation of the PZR towards the left can be argued from the slope.

Summarizing, we observe that all the materials show a range in which the potential rise is roughly linear in the 


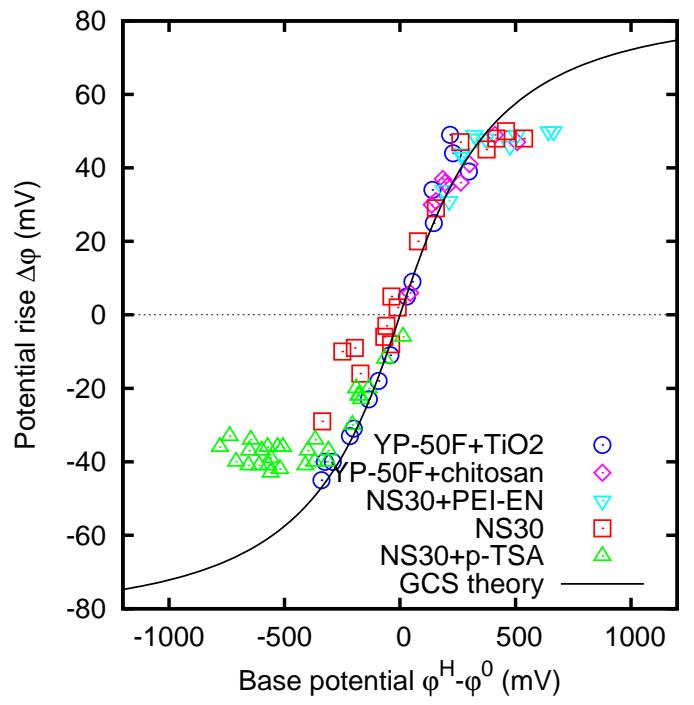

Figure 7: Experimentally measured potential rise $\Delta \varphi$ of the various activated carbon electrodes in the same conditions of Fig. 4. The various datasets are translated along the horizontal axis so that the zero-rise is at the origin. The line represents the result of GouyChapman-Stern theory with $C_{S t}=80 \mathrm{mF} / \mathrm{m}^{2}$.

base potential, with approximately the same slope. The adsorption of PEI-EN and chitosan (polymers with positive charges) decreases the $\mathrm{PZR} \varphi^{0}$, while adsorption of $\mathrm{p}$ TSA (a molecule with negative charge) increases the PZR. Moreover, the region in which the linear dependence is observed corresponds to a belt of $\Delta \phi$ values approximately ranging from -40 to $+50 \mathrm{mV}$; outside this region two saturations are observed, at approximately -40 or $+45 / 50 \mathrm{mV}$ respectively. It can be noticed that whenever these saturations were not experimentally observed, they are indeed expected to take place outside the window of observability of the base potential, which is limited by the redox reactions involving water.

In order to verify in a more direct way the fact that the slopes of the potential rise curves in the transition zone are similar for all the samples, we plot all the available data points together on the same graph, upon a translation such that the apparent PZR of each sample is shifted to the origin. Figure 7 shows the result of this procedure.

The graph shows that all the slopes are indeed similar; moreover, the saturations (when observable) take place at nearly the same values. The observation of this graph thus leads to the conjecture that all the potential rise versus base potential curves are roughly equal, up to a translation along the base potential axis. The horizontal displacement of the individual curves is apparently related to the presence of adsorbed charged molecules on the electrode's surface. In this way, for materials with different amounts of adsorbed charge, different portions of the same curve fall in the observable window. Hence we may think that the graph in Fig. 7 represents the reconstruction of a full potential rise versus base potential curve, over a wide range

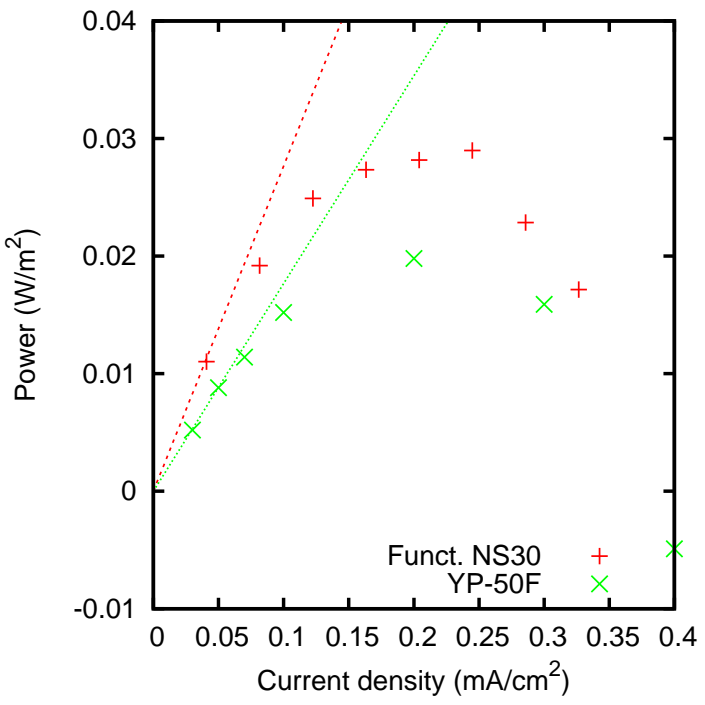

Figure 8: Power production from the CAPMIX cicle as a function of the current density, for NS30 films functionalized with p-TSA and PEI-EN, and YP-50F films with $\mathrm{TiO}_{2}$ and adsorbed chitosan. The straight lines correspond to $P=I \Delta V_{\text {cell }} / 3$.

of base potentials that for any individual sample is not accessible due to the instability of water.

Figure 7 also reports the theoretical prediction of the Gouy-Chapman-Stern (GCS) theory [38], with a Stern layer capacitance of $80 \mathrm{mF} / \mathrm{m}^{2}$. It can be observed that the data follow the GCS theory only around 0. For large absolute values of the base potentials, the potential rise reaches saturation levels which seem to show a slight asymmetry: the positive value is apparently close to $50 \mathrm{mV}$, while the negative one seems to be around $-40 \mathrm{mV}$. The GCS theory on the other hand predicts saturation levels at $\pm 83 \mathrm{mV}$, corresponding to the difference of chemical potentials of the ions in the two different concentrations, $20 \mathrm{mM}$ and $500 \mathrm{mM}$ respectively. This potential rise is indeed obtained in the CDP setup [23], since it also corresponds to the variation of the Donnan potential between the two concentrations. The saturations that we observe with our electrodes are much lower, about one half of these values. On the other hand, this means that we observe a plateau where the GCS theory still predicts a quite marked slope.

By performing CAPMIX cycles with electrodes made of functionalized carbon, we obtain results which are in agreement with the values of potential rise reported above. In Fig. 8 we show the produced power as a function of the current density, for CAPMIX cycles such that the duration of phases A and C (see section 1) was $240 \mathrm{~s}$, while that of phases $\mathrm{B}$ and $\mathrm{D}$ was $120 \mathrm{~s}$. One of the dataset refers to a cell with two electrodes made of NS30 activated carbon, one functionalized with p-TSA and the other with PEIEN. For the other dataset, the electrodes were made of YP$50 \mathrm{~F}$ activated carbon, and one of them was functionalized with chitosan.

It can be noticed that for low current densities, i.e. 
when the dissipative losses are negligible, the produced power asymptotically approaches the straight line $P=$ $I \Delta V_{\text {cell }} \eta$, where $\eta=1 / 3$ is the ratio between the length of phase $\mathrm{C}$ (the active phase) and the total duration of the cycle, and $\Delta V_{\text {cell }}$ is the cell voltage rise, which according to the data shown in Figs. 5 and 6 is approximately 83 $\mathrm{mV}$ for NS30 and $53 \mathrm{mV}$ for YP-50F. At higher currents, the effect of the internal resistance appears as a quadratic term which decreases the produced power.

\section{Discussion}

Various models of the electric double layer have been analyzed in the context of CAPMIX technique [31]. In this section we will apply them for explaining some of the features found in the described experiments.

The electrode of activated carbon material is modeled as a locally flat conductor, with a surface charge $\sigma$, dipped into a solution containing ions. We assume that the surface charge $\sigma$ can be changed by connecting the conductor to a power supply; moreover, we assume that $\sigma$ does not change when the conductor is isolated, i.e. we assume that the material can be treated as "polarizable", at least on the time scales of the seconds.

Two different charged layers can be identified in the solution close to the electrode surface. Following Ref. [38] we assume that, at a distance $\beta$ from the conductor surface, in the so-called "Stern layer", there is a flat charge distribution $\sigma^{i}$, that physically corresponds to a layer of permanently adsorbed charged molecules. At distances greater than $\beta$, the ions in the solution only interact electrostatically with the surface. Assuming that their distribution in space obeys Boltzmann's law, and using Poisson's equation to calculate the electric field in the solution, the GouyChapman-Stern theory provides an analytical expression for the charge distribution which is formed in the solution, as a function of the distance from the solid. This distribution represents the so-called "diffuse layer", and contains a total charge $\sigma^{d}[39]$ per unit area.

\subsection{Dependence of the potential of zero-rise on the charge density of the adsorbed molecules}

The effect of the presence of a charged Stern layer on the potential rise versus base potential graph, according to the Gouy-Chapman-Stern theory, is shown in Fig. 9. It can be clearly seen that the effect is a translation along the base potential axis [31].

It must be noticed that the base potential is experimentally measured with respect to a reference electrode, while the electrode potential in electric double layer theories is usually calculated with respect to the potential in the solution, at infinite distance from the electrode. The latter, absolute, potential is not actually accessible by measurements. For this reason, the "base potential" axis must be always considered as relative and arbitrary. Its zero simply represents the potential at which the electrode has

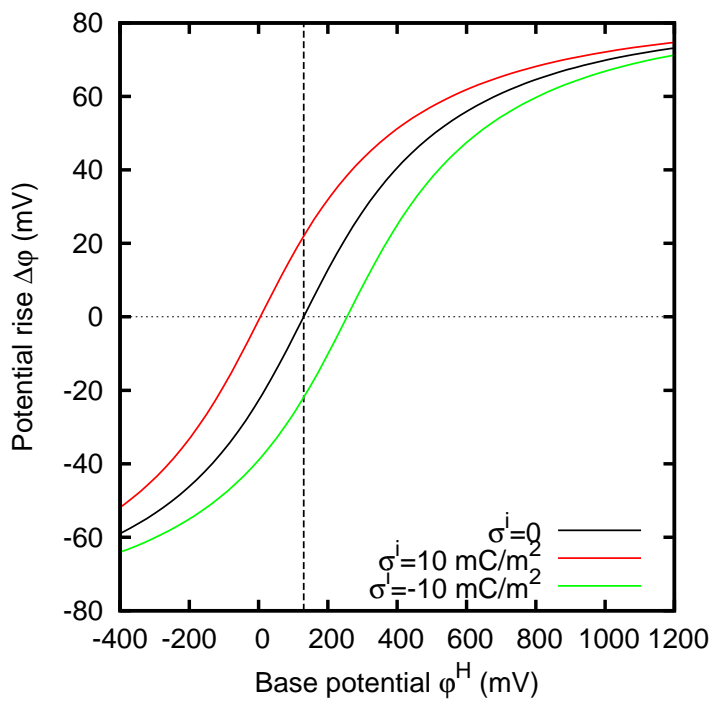

Figure 9: Calculation of the effect of a Stern layer with a fixed surface charge $\sigma^{i}$. Specific Stern layer capacitance: $C_{S t}=80 \mathrm{mF} / \mathrm{m}^{2}$. The potential is assumed to be measured with respect to a reference electrode, and its zero is arbitrarily set so that, when $\sigma^{i}=0$, the potential of zero rise is $130 \mathrm{mV}$.

\begin{tabular}{lll} 
Substrate & Adsorbed molecule & $\Delta \sigma^{i}\left(\mathrm{mC} / \mathrm{m}^{2}\right)$ \\
\hline NS30 & PEI-EN & +48 \\
NS30 & p-TSA & -46 \\
YP-50F & chitosan & +40
\end{tabular}

Table 1: Variation of the charge density produced by the adsorption of the charged molecules.

the same potential as a given reference electrode, and does not represent the situation in which the voltage across the electric double layer vanishes. In Fig. 9 we have chosen to set the 0 of the horizontal axis so that the PZR (potential of zero rise, i.e. the base potential at which the potential rise vanishes) of the uncharged sample is at $130 \mathrm{mV}$, as experimentally observed for NS30 with respect to a $\mathrm{Ag} / \mathrm{AgCl}$ reference electrode.

It can be noticed that the PZR $\varphi^{0}$ corresponds to the absence of the diffuse layer, i.e. $\sigma^{d}=0$. In this condition, the charge on the conductor is just opposite to the charge of the adsorbed molecules, i.e. $\sigma=-\sigma^{i}$. The effect of the adsorbed charged molecules on the PZR can be easily calculated. One finds that the difference $\Delta \varphi^{0}$ between the PZR of two carbon electrodes of the same material, such that their charge densities in the Stern layer differ by $\Delta \sigma^{i}$, is

$$
\Delta \varphi^{0}=-\frac{\beta}{\epsilon_{0} \epsilon_{r}} \Delta \sigma^{i}=-\frac{\Delta \sigma^{i}}{C_{S t}}
$$

where $C_{S t}$ is the capacitance of the Stern layer per unit surface. From the measurement of $\Delta \varphi^{0}$ it is possible to evaluate the variation $\Delta \sigma^{i}$ of the charge density of the adsorbed molecules with respect to the unmodified material. Results are reported in Tab. 1. 


\subsection{Saturation of the potential rise at high base potential}

Figure 7 shows that the potential rise saturates at about $50 \mathrm{mV}$ at high base potential, and at $-40 \mathrm{mV}$ at low potential. This saturation represents a discrepancy with respect to the Gouy-Chapman-Stern theory. This discrepancy cannot be explained in terms of non-ideality of the solution nor finite ion size, since the same effect would be also observed in the Donnan potential that develops across perm-selective membranes dipped into the $\mathrm{NaCl}$ solutions. In that case, instead, saturation at the full $80 \mathrm{mV}$ Donnan potential is observed [14] .

Another interesting comparison is with capacitive deionization (CDI) experiments. In particular, an accurate modeling of the experimental results, which was developed for CDI [40], predicts also in this case two plateaus at $\pm 80 \mathrm{mV}$ for the potential rise. It is worth noting that there is a thermodynamical relation [18] between the desalination charge efficiency of the above-mentioned models [40] and the potential rise of CAPMIX, that holds in equilibrium conditions. The apparent discrepancy that we observe between the capacitive deionization experiments and our CAPMIX potential rises should lead to the conclusion that the latter are not obtained in equilibrium conditions; indeed, they are measured on time scales that are shorter than that of the CDI experiments.

For the above reasons, we argue that the potential rise that we observe reaches a stationary value before the complete equilibration of the concentrations inside the activated carbon particles. We think that the pores are not equally bathed in the exchanging solutions all through the electrode volume. So, when the solution in contact with the beginning of the electrode changes to $20 \mathrm{mM}$, we cannot be sure that, even after a relatively long time, all pores are filled with the same solution. This means that the charge density is not the same all through the electrode, since the electric charge tends to accumulate in the regions where the capacity of the double layer is larger, and so in the parts of the electrode which are still in contact with a more concentrated solution. Thus only a model which takes these effects into account could provide a full explanation for the data represented in Fig. 7.

\section{Conclusions}

In the present paper we have investigated various types of activated carbons, which can be used for the realization of electrodes for the CAPMIX system [13]. In particular, we have focused on one of the most important properties which determine their performances for this application, namely the voltage rise. This was defined as the variation $\Delta \varphi$ of the electrode potential taking place when salt water is replaced by fresh water inside the CAPMIX cell. For each type of activated carbon we have studied the dependence of the potential rise on the base potential $\varphi^{H}$, defined in turn as the electrode potential in salt water with respect to a suitable reference electrode. The base potential can be arbitrarily varied to some extent by preliminarily charging the electrode with an external generator. In this way, for each sample a potential rise versus base potential curve was measured.

Suitable procedures in the preparation of the electrodes can introduce charged molecules which remain permanently attached to the surface of the activated carbon. We have investigated how the presence of such charged molecules affects the potential rise versus base potential curve. The main results are the following:

1. The adsorption of positive (negative) molecules displaces horizontally the curve towards lower (higher) base potentials.

2. Independently of the material and of the adsorption of molecules, the potential rise saturates to a value of about $+50 \mathrm{mV}(-40 \mathrm{mV})$ for very high (low) base potentials.

By analyzing the collected data, we have noticed that, if the potential rise is reported for each sample as a function of the difference $\varphi^{H}-\varphi^{0}$ between the base potential and the zero-potential-rise point, then the data for all the studied samples fall on a sort of "universal" curve. By means of the adsorption of charged molecules, we have been able to observe the behavior of this curve on a very wide range of values of $\varphi^{H}-\varphi^{0}$; this allowed us to clearly observe the saturation of the potential rise.

We have remarked that the result 1 , about the translation of the curve in presence of adsorbed charged molecules, can be explained using the Gouy-Chapman-Stern theory [31]. On the other hand, on the grounds of the same theory, one would expect values of about $\pm 80 \mathrm{mV}$ for the saturation levels of the potential rise. We have argued that the discrepancy between these figures and the observed values, as mentioned at point 2 above, can reasonably be attributed to kinetic effects. This hypothesis has however to be checked by further experimental and theoretical investigations.

From a practical point of view, the main outcome of this research is that the modification of the activated carbon, by means of the adsorption of charged molecules, allows us to obtain couples of electrodes giving a total cell voltage rise of about $90 \mathrm{mV}$, which is a quite satisfactory value for CAPMIX applications. Furthermore, this can be accomplished without the need of a preliminary external charging of the electrodes, which has typically the disadvantage of being accompanied by a slow but steady spontaneous self-discharge. For these reasons, the chemical modification of activated carbon electrodes appears to be one of the most promising techniques for the realization of efficient CAPMIX cells.

\section{Acknowledgments}

We thank M. Biesheuvel for useful discussions. 
The research leading to these results received funding from the European Union Seventh Framework Programme (FP7/2007-2013) under agreement no. 256868, CAPMIX project. LM acknowledges support of Cariplo Foundation Materiali Avanzati - 2011, Project 2011-0336.

[1] R. E. Pattle, Nature 174 (1954) 660.

[2] R. W. Norman, Science 186 (1974) 350-353.

[3] B. E. Logan, M. Elimelech, Nature 488 (2012) 313-319.

[4] J. Kuleszo, C. Kroeze, J. Post, B. M. Fekete, J. Integr. Environ. Sci. 7 (2010) 89-96.

[5] S. Loeb, Desalination 120 (1998) 247-262.

[6] M. Turek, B. Bandura, P. Dydo, Desalination 221 (2008) 462466.

[7] A. Cipollina, A. Misseri, G. D'Alí Staiti, A. Galia, G. Micale, O. Scialdone, Desalination and Water Treatment 49 (2012) 390403.

[8] O. Levenspiel, N. de Vevers, Science 183 (1974) 157-160.

[9] S. Loeb, Science 189 (1975) 654-655.

[10] T. S. Chung, X. Li, R. C. Ong, Q. G. H. Wang, G. Han, Curr. Opin. Chem. Eng. 1 (2012) 246-257.

[11] J. N. Weinstein, F. B. Leitz, Science 191 (1976) 557-559.

[12] J. W. Post, H. V. M. Hamelers, C. J. N. Buisman, Env. Sci. Techn. 42 (2008) 5785-5790.

[13] D. Brogioli, Phys. Rev. Lett. $103 \quad$ (2009) 058501. doi:10.1103/PhysRevLett.112.068103.

[14] B. B. Sales, M. Saakes, J. Post, C. J. N. Buisman, P. M. Biesheuvel, H. V. M. Hamelers, Env. Sci. Techn. 44 (2010) 5661. doi:10.1021/es100852a.

[15] D. Brogioli, R. Zhao, P. M. Biesheuvel, Energ. Environ. Sci. 4 (2011) 772-777. doi:10.1039/C0EE00524J.

[16] O. Burheim, B. Sales, O. Schaezle, F. Liu, H. V. M. Hamelers, Auto generative capacitive mixing of sea and river water by the use of membranes, ASME 2011 International Mechanical Engineering Congress and Exposition (IMECE2011) (Denver, Colorado, USA) p. 483-492, paper no. IMECE2011-63459, 2011. doi:10.1115/IMECE2011-63459.

[17] M. F. M. Bijmans, O. S. Burheim, M. Bryjak, A. Delgado, P. Hack, F. Mantegazza, S. Tennisson, H. V. M. Hamelers, Energy Procedia 20 (2012) 108-115. doi:10.1016/j.egypro.2012.03.013.

[18] R. A. Rica, R. Ziano, D. Salerno, F. Mantegazza, D. Brogioli, Phys. Rev. Lett. $109 \quad$ (2012) 156103. doi:10.1103/PhysRevLett.112.068103.

[19] R. A. Rica, R. Ziano, D. Salerno, F. Mantegazza, R. van Roij, D. Brogioli, Entropy 15 (2013) 1388-1407. doi:10.3390/e15041388.

[20] R. A. Rica, R. Ziano, D. Salerno, F. Mantegazza, M. Z. Bazant, D. Brogioli, Electrochimica Acta 92 (2013) 304-314. doi:10.1016/j.electacta.2013.01.063.

[21] R. A. Rica, D. Brogioli, R. Ziano, D. Salerno, F. Mantegazza, J. Phys. Chem. C 116 (2012) 16934-16938. doi:10.1021/jp3059849.

[22] B. B. Sales, F. Liu, O. Schaetzle, C. J. N. Buisman, H. V. M. Hamelers, Electrochimica Acta 86 (2012) 298-304. doi:10.1016/j.electacta.2012.05.069.

[23] F. Liu, O. Schaetzle, B. B. Sales, M. Saakes, C. J. N. Buisman, H. V. M. Hamelers, Energ. Environ. Sci. 5 (2012) 8642-8650. doi:10.1039/C2EE21548A.

[24] O. S. Burheim, F. Liu, B. B. Sales, O. Schaetzle, C. J. N. Buisman, H. M. Hamelers, J. Phys. Chem. C 116 (2012) 1920319210. doi:10.1021/jp306522g.

[25] B. B. Sales, O. S. Burheim, F. Liu, O. Schaetzle, C. J. N. Buisman, H. V. M. Hamelers, Environ. Sci. Technol. 46 (2012) 12203-12208. doi:10.1021/es302169c.

[26] H. V. M. Hamelers, O. Schaetzle, P. M. B. J. M. Paz-García C. J. N. Buisman, Environ. Sci. Technol. Lett. 1 (2014) 31-35. doi:10.1021/ez4000059.

[27] F. La Mantia, M. Pasta, H. D. Deshazer, B. E. Logan, Y. Cui, Nano Lett. 11 (2011) 1810-1813.

[28] Z. Jia, B. Wang, S. Song, Y. Fan, RSC Adv. 3 (2013) 2620526209. doi:10.1039/c3ra44902e.
[29] M. Marino, L. Misuri, A. Carati, D. Brogioli, Energies 7 (2014) 3664-3683. doi:10.3390/en7063664.

[30] M. Ye, M. Pasta, X. Xie, Y. Cui, C. S. Criddle, Energy Environ. Sci. 7 (2014) 2295-2300. doi:10.1039/C4EE01034E.

[31] D. Brogioli, R. Ziano, R. A. Rica, D. Salerno, F. Mantegazza, J. Coll. Interf. Sci. 407 (2013) 457-466. doi:10.1016/j.jcis.2013.06.050.

[32] D. Brogioli, R. Ziano, R. A. Rica, D. Salerno, O. Kozynchenko, H. V. M. Hamelers, F. Mantegazza, Energ. Environ. Sci. 5 (2012) 9870-9880. doi:10.1039/C2EE23036D.

[33] X. Gao, A. Omosebi, J. Landon, K. Liu, Electrochemistry Communications 39 (2014) 22.

[34] M. M. Goldin, V. A. Kolesnikov, M. S. Khubutiya, A. G. Volkov, G. J. Blanchard, A. K. Evseev, M. M. Goldin, J. Appl. Electrochem. 38 (2008) 1369-1374.

[35] S. Porada, R. Zhao, A. van der Wal, V. Presser, P. M. Biesheuvel, Prog. in Mat. Sci. 58 (2013) 1388-1442.

[36] Produced by Norit Activated Carbon, CABOT Inc., http://www.norit.com/.

[37] Produced by Kuraray Chemical Co., LTD, http://www.kuraraychemical.com/products/sc/capacitor.htm.

[38] J. Lyklema, Fundamentals of Interface and Colloid Science, volume 2, Academic press, New York, 1995.

[39] A. V. Delgado (Ed.), Interfacial Electrokinetics and Electrophoresis, Marcel Dekker, New York, 2002.

[40] P. M. Biesheuvel, S. Porada, M. Levi, M. Z. Bazant, J. Solid State Electrochem. 18 (2014) 1365-1376. doi:10.1007/s10008014-2383-5. 